\title{
Elaboración de un Modelo para Detectar y Corregir Impactos Ambientales Negativos del Turismo en la Comuna de Paihuano - Chile $^{1}$
}

Model Design for the Detection an Correction of Negative
Environmental Impacts of Tourism on the Paihuano Country - Chile Mauricio Alfaro ${ }^{2}$
Gabriel Canihuante
Angelo Ontaño

RESUMEN: Este documento presenta informaciones básicas del turismo en Chile y en la Región de Coquimbo y los avances de una investigación sobre el turismo que se desarrolla en Paihuano, comuna rural del centro norte de Chile. Dicha investigación se llevó a cabo entre abril 2001 y julio 2002 para la elaboración de un modelo que, a través de indicadores y parámetros, permita detectar y ayude a corregir los impactos negativos del turismo en esa comuna.

PALABRAS CLAVE: Chile; Región de Coquimbo; Comuna de Paihuano; Modelo; Indicadores de sustentabilidad turística; parámetros.
ABSTRACT: This paper presents basics informations about the tourism in Chile and the Coquimbo region and the advances of a research aboyt the 2003. tourism developped on Paihuano, a rural county of the north-center of

1. Resumen del informe de investigación presentado al Programa de Capacitación en Desarrollo Regional Sustentable, Universidad de La Serena, Chile. Este proyecto es financiado principalmente por entidades canadienses como CIDA (Canadian International Development Agency) y AUCC (Association of Universities and Colleges of Canada).

2. Mauricio Alfaro es ingeniero de sistemas y contador auditor. Ex director de la Escuela de Turismo ULS. Contacto: Alejandro Covarrubias, 6020 - La Serena, Chile; e-mail: mauricioalfaro@hotmail.com. 3. Gabriel Canihuante es periodista y magíster en Ciencias Geográficas. Académico ULS. Contacto: O’Higgins 655 - La Serena, Chile; e-mail: canimau@hotmail.com.

4. Angelo Montaño es egresado de la carrera de ingeniería comercial de la Universidad de La Serena y licenciado en Ciencias Administrativas. Contacto: Las Docas 4490 - La Serena, Chile; e-mail: am2e@hotmail.com. 
Chile. This research did between april 2001 and july 2002 and its objective was to do a Model that, through indicators and parameters, allow detect and help to correct the negativs effects the tourism on the county studied.

KEYWORDS: Chile; Region of Coquimbo; Paihuano county; model; indicators of turistic sustentability; parameters.

\section{Introducción}

En el marco del Programa Internacional de Capacitación en Desarrollo Regional Sustentable (DRS), llevado a cabo por las Universidades Nacional de Heredia (Costa Rica) y de La Serena (Chile) (1999-2003), dirigido por profesores de la University of Regina (Canadá), se inició en abril de 2001 la investigación denominada "Elaboración de un Modelo para la Medición y Corrección de Impactos Ambientales Provocados por el Turismo en la Comuna de Paihuano, Mediante la Generación de Estándares Relativos a Diversas Variables".

El resultado esperado para esta investigación era un modelo con el cual podrían pasar a operar las autoridades de la comuna de Paihuano para detectar a tiempo los impactos ambientales negativos del turismo en las diversas localidades que conforman la comuna. Se trataría de un Modelo fácilmente operativo, de modo que funcionarios de diversos sectores (Salud, Seguridad, Turismo y otros) y líderes de organizaciones de base, pudieran participar abasteciendo el sistema de información y, en algunos casos, tomando decisiones a partir de esa información.

La opción de trabajar sobre el turismo se debe a que, de acuerdo a las autoridades locales, este sector se ha convertido en los últimos años en la segunda actividad económica de importancia, luego de la agricultura, ocupación tradicional para la comuna. El turismo que se ha desarrollado con importancia económica para los residentes desde la década de los 90 , tiene como principal oferta para los visitantes el atractivo natural de la zona.

El carácter ecológico del turismo masivo que se registró en Paihuano en la segunda mitad de la década anterior, ha sido cuestionado por diversos actores. Como un aporte a la preocupación de residentes y autoridades de Paihuano, los autores de la presente investigación han orientado el curso de la misma hacia la elaboración de un instrumento que sirva en la toma de decisiones para reconducir el turismo hacia un desarrollo de tipo sustentable.

\section{Metodología}

Para esta investigación, exploratoria, descriptiva y propositiva, sus autores trabajaron con diversas fuentes y establecieron una serie de pasos:

a) información documental;

b) trabajo de campo: visitas a terreno en diferentes épocas; aplicación de encuestas a residentes; tabulación y análisis posterior; entrevistas a personas clave y análisis de los resultados; recogida de datos específicos (caso de Salud); selección de información a partir de otras instancias de interés;

c) reuniones de evaluación para la determinación de las variables principales a considerar en el Modelo (componentes ambientales e impactos), así como el peso relativo (ponderaciones) de estos últimos;

d) elaboración del informe de investigación.

\section{Marco Referencial}

El turismo como muchas otras actividades económicas y sociales implica una serie de impactos en el ambiente. El turismo depende casi absolutamente del ambiente, del entorno en que se desarrolla. Si hay un ambiente contaminado que afecta a un atractivo (sea urbano, rural, de montaña o costero), el turista dejará de sentir atracción por ese lugar y más temprano que tarde terminará desplazándose a otro destino. El consumo del producto / servicio turístico es consustancial a su entorno; el turista consume aire puro, paisaje, tranquilidad y otras características $\mathrm{y}$ atributos impregnados en el medio.

En los últimos años, los consumidores de los países desarrollados están demostrando una preocupación creciente por los problemas ambientales. Así los destinos turísticos que sufrieron en su día un desarrollo espontáneo y desordenado y sin ninguna consideración hacia el medio ambiente, en términos de conservación y mejora, están viendo descender sus entradas de visitantes, debido a los cambios en las preferencias de los consumidores y a la aparición de competidores con entornos no degradados. (OMT, 2001: 254)

El turismo puede ser una actividad con resultados positivos o negativos para una localidad, ciudad o país, según se desarrolle en forma planificada o no. El turismo espontáneo que crece ocupando áreas geográficas en función exclusivamente del aumento de las ganancias de las personas, empresas y Estados involucrados, tiende a traer a mediano y largo plazos efectos tan negativos que inciden en una baja de la calidad del atractivo. 
(...) si el turismo no está bien planificado, desarrollado y gestionado, puede ser la causa de congestión, contaminación y otros problemas ambientales, así como de importantes disfunciones en la sociedad en la que interactúa. (OMT, 2001: 257)

La planificación implica postular un desarrollo turístico limitado, con la búsqueda de un punto de equilibrio entre lo que los visitantes quieren (fuerza de la demanda) y lo que el lugar puede soportar sin sufrir efectos irreversibles o insoportables. Podemos también señalar que este tipo de turismo debe ser sustentable, el que según definición de la O.M.T. es aquel que:

(...) atiende a las necesidades de los turistas actuales y de las regiones receptoras y al mismo tiempo protege y fomenta las oportunidades para el futuro. Se concibe como una vía hacia la gestión de todos los recursos de forma que puedan satisfacerselas necesidades económicas, sociales y estéticas, respetando al mismo tiempo la integridad cultural, los procesos ecológicos esenciales, la diversidad biológica y los sistemas que sostienen la vida. (OMT, 1999: 22)

Para Rivas (1998), "la sustentabilidad de una zona turística depende de un conjunto de factores ambientales, sociales, culturales y económicos que involucran tanto a la industria turística propiamente tal, como la comunidad residente y a los propios turistas". Fayos Solá, citado por Rivas, señala que "a partir de la consolidación de lo que se ha denominado la Nueva Era del Turismo (NET), donde predomina la motivación por participar de experiencias recreativas vinculadas al medio ambiente, y cuando la calidad constituye un factor determinante en la valoración que se le asigna a los productos turísticos, puede afirmarse que el objetivo de alcanzar un desarrollo sostenible no sólo tendrá un efecto positivo en el ámbito ecológico y socio cultural, sino que en muchas zonas, y principalmente en el entorno rural, será una condición básica para asegurar la presencia del turismo como actividad económica”. (Rivas, 1998: 48)

La preocupación por anticipar y prevenir efectos no deseables de la actividad turística de parte de la OMT se manifestó hace varios años (desde 1995/96) y el resultado de un proyecto específico relacionado con el tema es la publicación, en 1997, de la Guía Práctica para el Desarrollo y Uso de Indicadores de Turismo Sostenible".

El texto de la OMT (1999: 10) aclara que:

Debe tenerse en cuenta que los indicadores sociales y medioambientales no son frecuentemente susceptibles de evaluación económica. ... Debido a ello, el valor de las funciones ecológicas puede estar constantemente subestimado en los modelos económicos y de contabilidad tradicionales y, consecuentemente, por las instancias decisorias. Por esta razón, los indicadores de sostenibilidad no siempre son cuantificables y pueden ser, necesariamente, algo subjetivos. Esta limitación, sin embargo, no supone ningún menoscabo para su utilidad como información para la gestión en la promoción del turismo sostenible.

Uno de los conceptos clave en el manejo de las zonas turísticas y requisito indispensable para la realización de un turismo sustentable es el de Capacidad de carga, tratado por diversos autores, entre ellos Manning (1998).

En el caso de Chile, el Gobierno, mediante la Política Nacional de Turismo, ha manifestado su preocupación respecto de la sustentabilidad del turismo y el necesario equilibrio entre los intereses privados y el bien público:

El turismo es una actividad que se ejerce y depende de la generación, preservación y desarrollo de bienes públicos, sean estos naturales, históricos y culturales. Es decir, se trata de la combinación de una actividad privada que atrae numerosos demandantes que presionan sobre la oferta de bienes públicos. Ello puede generar la emergencia de conductas que tiendan a degradar, destruir y desaprovechar atractivos y recursos turísticos, con lo cual la vida turística en aquellos lugares sería efímera o perdería la calidad necesaria para mantener la rentabilidad de las inversiones. También, por ausencia de normas o regulaciones incorrectas o permisivas, se inutilizan lugares con importantes atractivos turísticos, al destinarlos a otro tipo de actividades que no son compatibles con el desarrollo del turismo. (Sernatur, 1998: 5)

En cuanto a los impactos ambientales generados por la actividad turística identificados en Chile, se conoce un cuadro de ellos elaborado por Rivas (1998), a partir de informaciones entregadas por Sernatur, en el cual se señalan once componentes ambientales y un total de 85 diversos impactos.

\section{La Búsqueda de un Modelo Adecuado}

Una primera recomendación es la de comenzar siempre con modelos sencillos y que luego se vayan completando. Consejo éste que parece plenamente válido dada la complejidad del sistema turístico para adaptarlo a un modelo. Dado un sistema turístico, construir un modelo (aunque sólo sea para un aspecto de la actividad) "supone renunciar a reproducir algunos de los elementos que componen el sistema y a algunas de las relaciones o interacciones entre esos elementos". 
Los dos fines básicos del proceso de modelado son: primero, reproducir el comportamiento histórico (pasado) de los principales elementos (variables) del sistema; Segundo, disponer de un procedimiento con qué simular el comportamiento futuro de dicho sistema, ante supuestos alternativos. (Castillo y González, 2001: 7)

\section{Tasas o parámetros}

$\mathrm{Al}$ enfrentar la necesidad de un Modelo podemos estar ante un problema estructurado o semi-estructurado. Este último tipo de problemas puede ser analizado en subproblemas de tipo estructurados y no estructurados. De acuerdo a nuestro criterio, el problema del turismo sustentable en Paihuano es un problema de tipo semi-estructurado y la búsqueda del Modelo se hará en esa dirección.

\section{Modelos}

Un modelo es una representación o una abstracción de la realidad. El objetivo de un modelo es representar fielmente un problema y que a la vez presente una visión simplificada sobre él, lo que resulta en la práctica muy difícil de conseguir.

En nuestra investigación el modelo que utilizaremos será de tipo matemático, por ser el problema de alta complejidad y así permitir el análisis de un mayor número de soluciones, además de permitir el estado de las variables y evitando las malas interpretaciones, con un apoyo del modelo de tipo analógico en su presentación.

Componentes del modelo matemático. Todo modelo matemático consta de tres componentes: parámetros y variables no controladas, que afectan el resultado del proceso y sobre las cuales es imposible actuar; variables de decisión controladas, cuyo control depende de quien modela y que pueden ser alteradas, ya que se tiene el control; y por último variables resultantes, que reflejan el nivel de eficiencia del modelo. Los resultados, por lo tanto, son función de una serie de variables que se controlan y de otras variables que no pueden ser controladas. Posteriormente, los componentes de los modelos matemáticos, se estructuran y unen mediante expresiones matemáticas.

\section{El proceso de modelado}

Existen variadas técnicas para llegar a conocer el problema que queremos resolver, entre estas se encuentran: el ensayo, la simulación de ensayos, y la
Optimización. Nuestro trabajo se basará en la optimización generando un software de tipo modular que podrá ser utilizado por los diversos usuarios involucrados en el proceso de información y toma de decisiones.

En este proceso de modelado se han tenido en cuenta diversos elementos, que por razones de espacio no se detallan en este resumen. Son elementos tales como:

- criterios de evaluación;

- desarrollo de las alternativas;

- predicción de los resultados;

- medida de los resultados;

- selección de la opción.

\section{Resultados de la Investigación}

\section{El turismo en Chile y en Coquimbo}

De acuerdo a las últimas estadísticas de Sernatur, en 2001, Chile recibió a 1.721.709 turistas extranjeros, con una leve baja con relación a 2000, cuando llegaron al país 1.742 .407 visitantes de otros países. En términos de ingresos, en 2000, el turismo extranjero generó divisas por un total de 826,3 millones de dólares, cifra inferior en $7,9 \%$ respecto de 1999 .

El turismo generó el 4,1 \% del Producto Interno Bruto en 1996, según un estudio de Cuenta Satelital, investigación de carácter pionero realizada por Sernatur, Dirección Nacional, y cuyos datos parciales fueron divulgados en octubre de 1999. De acuerdo con ese estudio, el turismo es el quinto sector en aporte al PIB, situado luego de la industria manufacturera $(20,4 \%)$; servicios financieros (12\%); la minería $(7,5 \%)$ y el área agropecuario silvícola $(5,7 \%)$.

El turismo es una de las cuatro principales actividades económicas de la Región de Coquimbo, junto a la agricultura, la minería y la pesca. Esta Región se sitúa a 193 kilómetros al norte de Santiago y se extiende entre los $29^{\circ} 02^{\prime}$ y $32^{\circ} 16^{\prime}$ de latitud sur y desde los 69० 49'de longitud oeste hasta el Océano Pacífico. La población total de la región de Coquimbo, según datos preliminares del Censo oficial (INE, 2002), es de 600.363 habitantes, de los cuales el 70\% es urbana, mayoritariamente asentada en la conurbación La Serena-Coquimbo. La Región ocupa una superficie de $40.656,3 \mathrm{~km}^{2}$.

Para la Región de Coquimbo, el turismo como manifestación masiva es una actividad de interés relativamente nueva, a partir de la década de los 70 , cuando 
se generan flujos crecientes de visitantes atraídos por un turismo de "sol y playa", una actividad temporal, que dura el verano austral (diciembre a febrero).

Según el Estudio del Gasto Turístico (Sernatur, 1999a), se reportaron ingresos por 53,8 millones de dólares entre marzo de 1998 y febrero de 1999, cifra que se refiere básicamente a los ingresos generados por establecimientos de alojamiento y, por proyección, a los gastos realizados por personas que vacacionaron en esa zona en servicios como alimentación, transporte y recreación. La Región de Coquimbo representa el 5,3 \% de los ingresos nacionales en turismo receptivo, de acuerdo con la misma fuente.

\section{Algunos datos de la comuna de Paihuano}

La comuna de Paihuano se ubica a 90 kilómetros al sur-oriente de La Serena, limita en su parte oriental con Argentina. Tiene una superficie de $1.716 \mathrm{~km}^{2}$, lo que representa un 4,2\% de la superficie regional (Pladeco, 2000), y una población de 4.417 habitantes (INE, 2002). Por primera vez, el Censo oficial registra una población urbana en esta comuna. Hasta ahora se consideraba a toda la población como de carácter rural. Es relevante que este Censo mostró que se revirtió la tendencia decreciente de la población en los últimos decenios.

La principal actividad económica es la agricultura, con producción de frutas (uva de mesa y para producir pisco, kivis, duraznos, damascos y otras). El otro sector productivo es el silvoagropecuario.

\section{El desarrollo del turismo en Paihuano}

De las diversas formas de obtención de informaciones y datos sobre el turismo en Paihuano presentamos a continuación resúmenes de las principales ideas extraídas a lo largo de esta parte de la investigación, que se extendió desde abril de 2001 hasta febrero de 2002.

\section{a) Observaciones en terreno}

Al analizar los registros hechos en dos fechas distintas como el 15/01/02 (temporada alta) y el 24/08/02 (temporada baja), y según la percepción de los observadores (los tres investigadores), en resumen, podemos señalar que, de acuerdo a las visitas efectuadas, no existen grandes diferencias con lo que sucede a diario en la comuna. No se observó saturación en los servicios, aunque se destaca la mayor presencia de personas en Verano por lo que significa la temporada estival. b) Las encuestas

En el mes de octubre de 2001, se aplicó una encuesta de manera proporcional al número de habitantes de las tres localidades en estudio: Paihuano, Pisco Elqui y Horcón (una muestra de 143 habitantes) elegidos aleatoriamente.

Sobre el $92 \%$ de los habitantes considera bueno o muy bueno el lugar donde vive, lo que demuestra una fuerte identidad y valorización de su comuna, y consideran como una de las principales ventajas la tranquilidad (71\%), el clima(19\%) y la buena relación y cordialidad con los vecinos (15\%), y las principales desventajas son el desorden en verano, la falta de comunicación y servicios turísticos, pavimentación y la contaminación.

Sobre el $76 \%$ de los encuestados dijo que ve el turismo como positivo y un $17 \%$ lo veía como negativo. La percepción futura sobre el turismo es positiva en un $91 \%$. Lo positivo que trae el turismo está principalmente en que genera beneficios económicos en ventas, ingresos, etc. $(26,57 \%)$; genera empleo (15\%) y se conoce a gente y se aprende de ellos (12\%).

Lo que más molesta del turismo es la presencia de "mochileros" (en general, jóvenes de pocos ingresos que practican "turismo" de campamento salvaje) que se les cataloga hasta de gente indeseable y se propone hasta su prohibición de ingreso (31\%), la contaminación que gencran los visitantes especialmente en las riberas de ríos $(25,17 \%)$ y un $22 \%$ el desorden de verano.

Cuando se le consulta si, a futuro, le gustaría trabajar en el turismo, el 78,32\% manifiesta que sí le gustaría, principalmente en alojamiento, alimentación y guías.

c) Datos sobre atención de salud

Para tener una idea acerca del impacto específico que causa el turismo en los servicios de Paihuano, optamos por conocer datos del Servicio de Salud, ya que nos habían manifestado que se trataba de un impacto fuerte. En el Policlínico de Paihuano, se revisaron los registros de las consultas de las tres localidades en estudio (más la de Montegrande) durante un periodo de tres semanas compuestas (12.11.2001 al 21.04.2002).

El principal dato que nos interesaba conocer era la cantidad total de atenciones y qué porcentual de ese total era de turistas, ló que se puede detectar porque en el registro se anota la procedencia de la persona atendida. Asumimos que toda persona que no era de la comuna de Paihuano era un visitante o turista. El resultado numérico no fue realmente significativo, no se observó un cambio drástico entre Temporada Alta y Temporada Baja, de modo que no puede decirse, según estos datos, que el turismo provoque un impacto negativo considerable en este servicio. 
d) Taller de líderes comunitarios

En un taller efectuado en octubre de 2001, se reunieron 32 líderes comunitarios representativos de diferentes organizaciones sociales y de nueve localidades de la comuna, quienes llevaron a cabo un análisis DFOA (Debilidades, Fortalezas, Oportunidades y Amenazas) de sus localidades y de sus organizaciones de base. En todos los grupos de trabajo, estuvo presente el turismo. Las conclusiones obtenidas en función del turismo (omitidas, en esta versión, por razones de espacio) muestran que hay, entre los líderes comunitarios, conciencia del entorno (aspectos positivos y negativos) en que viven y conocimiento de la importancia del turismo como actividad económica, con sus ventajas y desventajas.

\section{Otros antecedentes}

Respecto del desarrollo del turismo en Paihuano, es conveniente registrar otras informaciones de interés obtenidas de diversos documentos y fuentes.

La afluencia masiva de turistas en Paihuano se registra desde los años 90 y es el resultado de un fenómeno de expansión típico de destinos con capacidad de distribución, como La Serena y Coquimbo. El Valle de Elqui - que se extiende por una parte de la comuna de La Serena, Vicuña y Paihuano - funciona como área de influencia.

Según el Departamento de Turismo de la Municipalidad de Paihuano, se estima que los visitantes a la comuna en 1997 alcanzaron a 70 mil, mientras que, en 1999/2000, llegaron a los 300 mil. Datos más recientes señalan que, en el verano de 2000-2001, llegó a la comuna un total de 150 a 200 mil visitantes, mientras que, para el verano de 2001-2002, hubo un registro de 4.616 visitantes por día*. Si entendemos que una temporada veraniega tiene 60 días (considerando solamente los meses de enero y febrero, que son los de mayor demanda), esto significa que hubo una llegada de cerca de 280.000 personas.

En 1996, se creó el Departamento Municipal de Turismo y, en 1997, se elaboró el Plan de Desarrollo Turístico de la Comuna (PDTC) de Paihuano. Según ese Plan, la comuna contaba, en términos de infraestructura turística, en 1996, con 13 establecimientos de alojamiento turístico y 14 establecimientos de alimentación (1108 sillas). En el año 2000, otro estudio (Campos, 2000) daba cuenta de la existencia de 36 establecimientos de servicio turístico (alojamiento,

\footnotetext{
* Registro tomado el miércoles 13 de febrero de 2002, Comunicación personal con Verónica Salazar, Encargada de Turismo en la Municipalidad de Paihuano, 2002.
}

alimentación y otros servicios). Es decir, en un periodo de cuatro años, hubo un aumento de $33 \%$ en establecimientos legales de servicios turísticos.

El PDTC señalaba que la motivación principal estaba centrada en los aspectos naturales de la comuna, lo que sigue vigente. Se afirmaba, en 1997, que "durante la temporada estival, se produce un problema de saturación en la mayor parte de los establecimientos que ofrecen servicios turísticos...".

Existe, desde 1999, una "Asociación Gremial de Empresarios Ecoturísticos de la Comuna de Paihuano", formada por 32 empresarios locales. Paralelamente, está en ejecución un proyecto de turismo rural, con apoyo de organizaciones estatales y financiamiento internacional.

Según diversas fuentes (prensa local, autoridades y los autores de este estudio), una cantidad no precisada de visitantes en verano está compuesta por jóvenes campistas que buscan cualquier tipo de sitios de campamento, incluyendo las riberas de los ríos de Paihuano no habilitadas para ese tipo de actividad. "Entre 2.000 y 3.000 personas se quedan en campings ilegales y en lugares no autorizados para acampar dentro de la comuna de Paihuano. Esto, obviamente, trae consigo varios problemas", señala un documento de trabajo de la Municipalidad (Análisis y estadísticas, 2000).

Esta situación tiende a cambiar a partir del verano 2001-2002, cuando se implementó un programa especial de control de visitantes. El proyecto GuardaRío, que fiscalizó la entrada de turistas y visitantes en un sector de la comuna durante ocho semanas, es parte de la Comisión de Turismo, Ambiente y Seguridad (Cotase), formada por funcionarios municipales de Vicuña y Paihuano.

\section{Modelo de Simulación del Impacto del Turismo para Paihuano}

El modelo pretende entregar, de manera simplificada, la información referente a los diversos componentes ambientales asociados a los impactos del turismo en éstos, con el fin de permitir una optimización de la toma de decisiones respectivas.

\section{Características del modelo}

- Modelo Matemático: el modelo tiene como base de realización la conformación de relaciones matemáticas entre diversos componentes ambientales, sus impactos y la correlación que sobre ellos tiene el número de turistas. 
- Gráfica: las relaciones establecidas permitirán al modelo presentar gráficamente la situación simulada de un determinado componente o sus impactos, permitiendo la evaluación en los diferentes niveles de actividad posibles de una manera fácil y amigable.

- Aproximación: el modelo entrega una aproximación a la realidad, por lo que utiliza valores cuantificables y niveles máximos estandarizados, así como valores de acuerdo a la percepción de las fuentes de información, en donde no existe procedimiento, instrumental o no está disponible para su utilización.

- Modular: el modelo permite la visión de los impactos del turismo, tanto en su perspectiva global como por componente ambiental, permitiendo el trabajo en módulos, que se pueden agrupar de manera conveniente - dependiendo del usuario y de la información requerida para la toma de decisión.

- Predictivo: el modelo permitirá predecir los efectos que provocará el turismo en las diversas variables ambientales permitiendo tomar medidas no sólo de carácter correctivo, sino además de carácter preventivo.

- Fácil uso: el modelo se presenta en una planilla electrónica en que se debe llenar solo el campo de número de turistas para obtener la simulación total de los impactos que el turismo provoca.

- Suministro de información: para la manutención de la operatividad del sistema, se hace necesaria la actualización de la valoración de los impactos, la que se puede conseguir de una manera cuantitativa, a través de mediciones específicas realizadas por los organismos especializados que corresponda (por ejemplo, la Empresa de Servicios Sanitarios de Coquimbo - ESSCO - para la Componente Agua), o a través de entradas cualitativas proporcionadas por organismos comunitarios, como juntas de vecinos, entre otros. Para estos últimos deben construirse (segunda etapa de esta investigación) algunos instrumentos específicos de medición, como fichas o encuestas.

\section{Metodología de construcción de modelo}

Detección de componentes e impactos: para la elaboración del modelo, el equipo de investigación debió realizar reiteradas salidas a terreno para detectar los componentes ambientales y los principales impactos que provoca el turismo en la comuna. Asociada esta información a la recogida en las diferentes fuentes bibliográficas, se llegó a determinar y delimitar nueve componentes y 39 impactos relevantes a utilizar.

Clasificación: luego de haber determinado los componentes e impactos, se procedió a la clasificación en problemas estructurados y no estructurados, con el fin de reconocer la mejor manera de enfrentar la problemática.

Determinación de relaciones: una vez identificados los componentes, sus impactos y su relación con el número de turistas se procedió a la estructuración de relaciones matemáticas a través de funciones que reflejaran el comportamiento de las tendencias de los impactos. Para ello, se elaboraron ponderadores, tanto para cada componente como para sus impactos asociados. El procedimiento de elaboración de ponderadores consistió en evaluar, con puntajes de uno a siete, la importancia de los impactos en forma individual por cada componente, con el fin de determinar la importancia relativa de estos (tomando como nota siete lo que más impacta y nota uno lo que no impacta). Una vez determinado el nivel o relevancia relativa de los impactos, se realizó un proceso similar con los componentes, es decir de acuerdo a su valoración, establecida por los puntajes antes señalados, se procedió a la determinación de la importancia relativa dentro del total de componentes, asignándole, en ambos casos, valores porcentuales a la importancia relativa.

En esta etapa, además, se determinó el efecto por niveles de actividad, considerando como agente de impacto al turista, es decir, se determinaron diez intervalos con un aumento de 500 turistas en cada uno de ellos, partiendo el primero de cero a 500 turistas, para finalizar con el intervalo de 4.500 a 5.000 turistas. Para esta fase, se tuvo en cuenta que la población local bordea los 4.000 habitantes.

Una vez determinado el efecto en forma cuantitativa, sobre la base de estimaciones de los investigadores, se procedió a su representación mediante una función ajustada, la que permite la simulación en cualquier nivel de actividad. También se determinaron, por parte de los investigadores, los niveles máximos aceptables para considerar a la actividad turística como sustentable.

Cabe destacar que, en esta primera fase de desarrollo del modelo, la valoración de los impactos fue realizada de una manera subjetiva por parte de los investigadores, para, en una segunda etapa, cuantificar de acuerdo con los organismos especializados para cada impacto.

Procedimiento de evaluación: el modelo considera una etapa de evaluación durante la cual se determina, de manera porcentual, cuál es el grado de valoración y éste se relaciona con el nivel máximo aceptable. 
Representación gráfica: el modelo considera la presentación de sus resultados en un software de fácil utilización (a modo de ejemplo, se ha utilizado Excel) en donde se realizan las representaciones gráficas del modelo en general y de sus componentes, accediendo, si fuese necesario, a los diversos componentes en forma individual y a sus respectivos impactos.

\section{Representación gráfica del modelo}

A continuación, se presentan algunas de las relaciones entre componentes que conforman el modelo, partiendo por el cuadro general del mismo con sus nueve componentes (Figura 1):

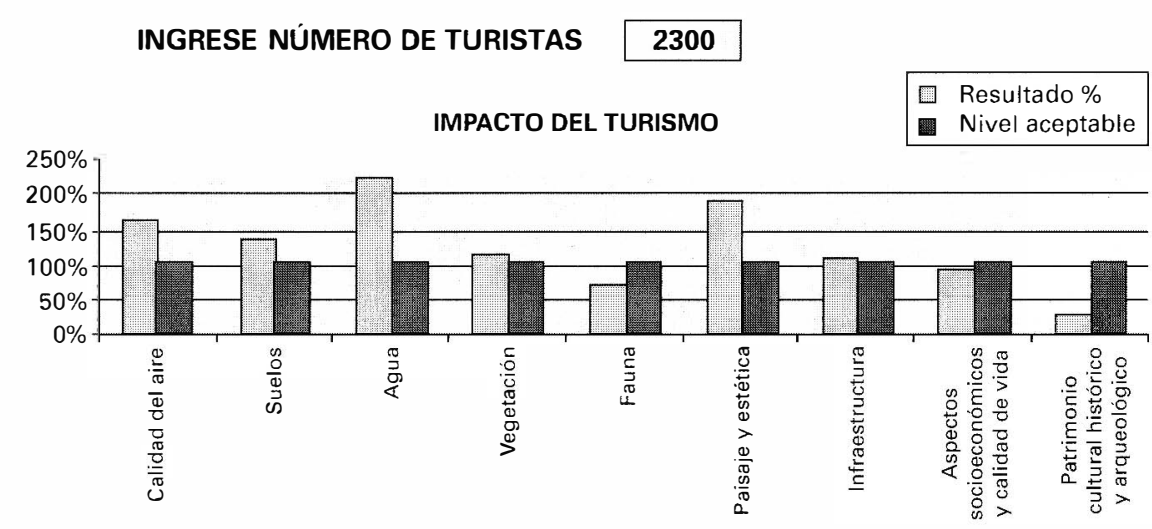

SI DESEA VER MÁS DETALLE SELECCIONE LA OPCIÓN:

\begin{tabular}{|c|c|}
\hline IDAD DEL AIRE & ASPECTOS SOCIOECONÓMICOS \\
\hline$\frac{\text { SUELOS }}{\text { AGUA }}$ & YCALIDAD DE VIDA \\
\hline VEGETACIÓN & PATRIMONIO CULTURAL \\
\hline $\begin{array}{l}\text { FAUNA } \\
\text { PAISAJE YESTÉTICA }\end{array}$ & HISTÓRICOY \\
\hline INFRAESTRUCTURA & ARQUEOLÓGICO \\
\hline
\end{tabular}

Figura 1. Relaciones entre componentes que conforman el modelo, partiendo por el cuadro general del mismo con sus nueve componentes

Los nueve componentes sobre los que se basa el modelo son: calidad del aire; suelos; agua; vegetación; fauna; paisaje y estética; infraestructura; aspectos socioeconómicos y calidad de vida; patrimonio cultural histórico y arqueológicos. Los que se desglosan en diversos impactos.
Presentación de las relaciones simuladas de los impactos de los componentes calidad del aire (Figura 2), fauna (Figura 3) y paisaje y estética (Figura 4):

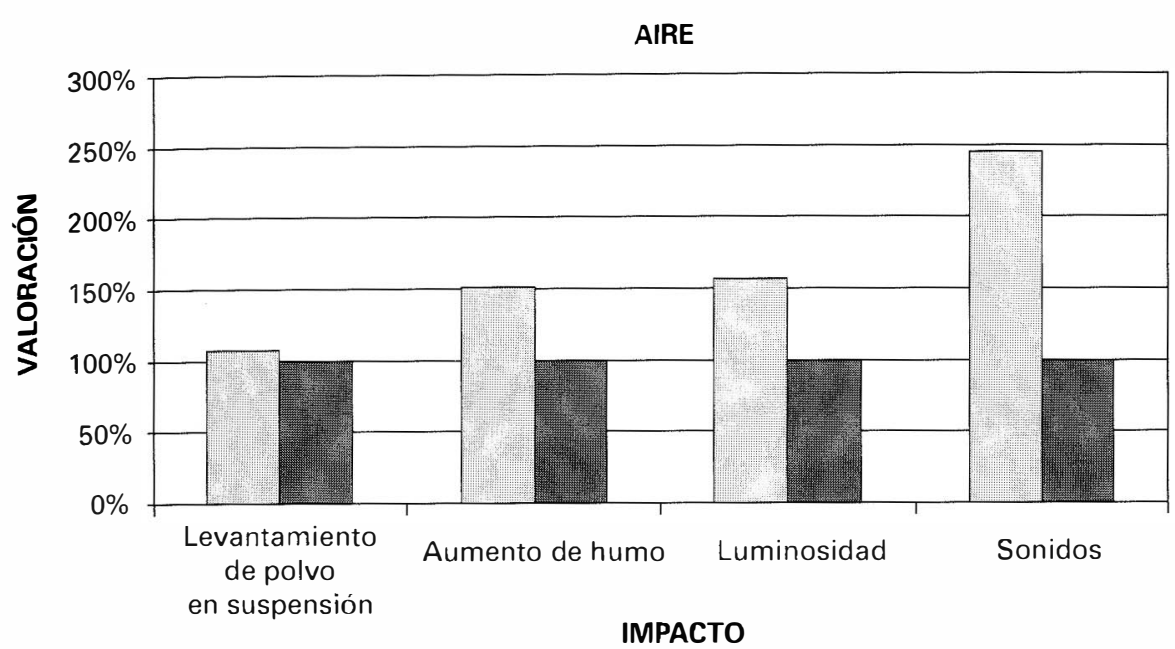

Figura 2. Relaciones simuladas de los impactos del componente calidad del aire

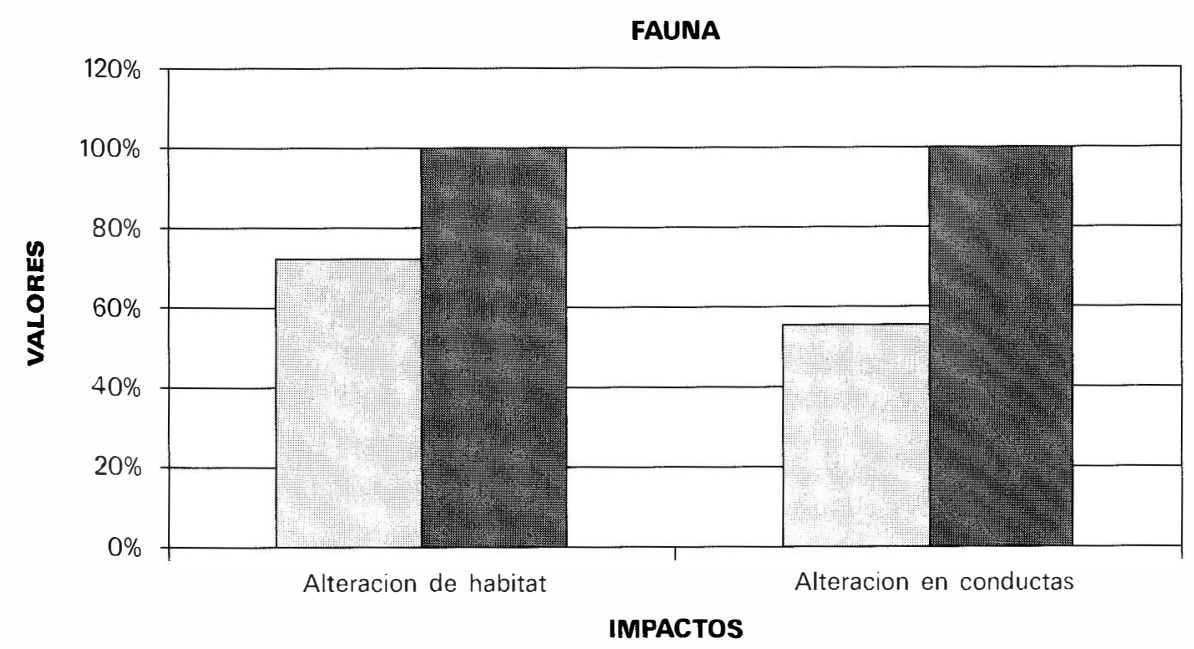

Figura 3. Relaciones simuladas de los impactos del componente calidad del aire 
PAISAJE Y ESTÉTICA

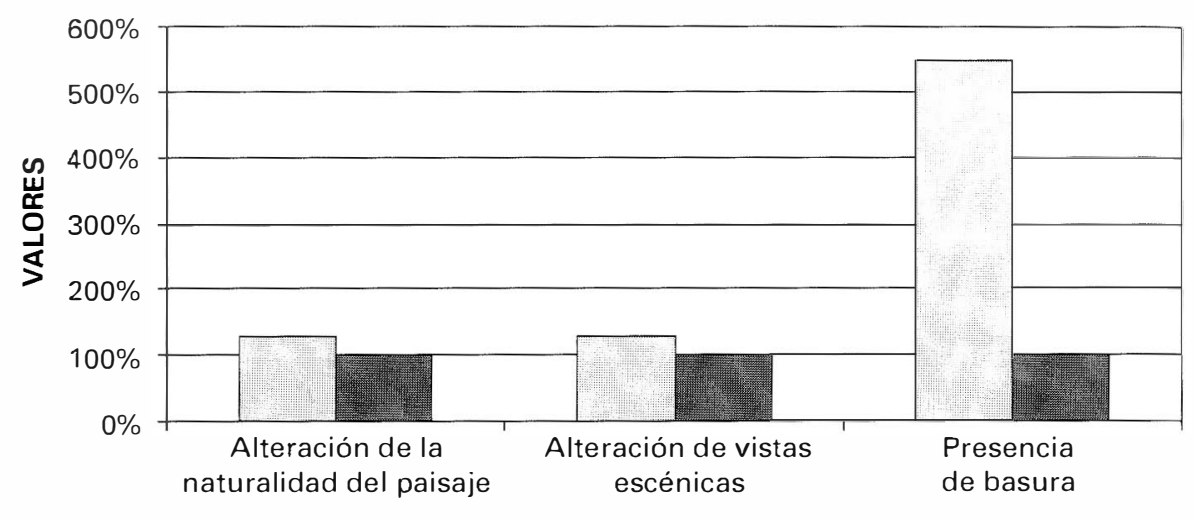

IMPACTOS

Figura 4. Relaciones simuladas de los impactos del componente paisaje y estética

\section{Conclusiones}

Una primera cuestión que nos interesa aclarar se refiere a que esta investigación no ha sido terminada; lo que aquí se presenta es un informe de avance. En esta etapa, hemos podido avanzar en términos de conocer profundamente el área de estudio, el tipo de turismo que allí se desarrolla, la percepción de los residentes respecto del turismo y la preocupación de autoridades y residentes respecto del futuro inmediato de esta actividad.

Además, determinamos un modelo para medir los impactos del turismo en Paihuano. En este modelo, se hizo una simplificación del sistema en estudio y se llegó a fijar un total de nueve componentes ambientales y un total de 39 impactos o efectos. Como no se pudo disponer, hasta ahora, de la información específica sobre estas variables, para probar nuestro modelo, hicimos una estimación cuantitativa de cada uno los impactos en función del cambio en la cantidad de visitantes y turistas en Paihuano.

Lo que presentamos es un modelo con datos estimados, y no reales. En la segunda fase, debemos incorporar esa información real al modelo para ver cómo se comporta el mismo y establecer modificaciones si fuera preciso.

En otro plano, a partir de nuestra observación e investigación en estos 16 meses (abril de 2001 a julio 2002), hemos podido concluir lo siguiente: a) si bien la comuna de Paihuano posee condiciones naturales y culturales de gran atractivo que permiten ofrecer turismo rural, y aunque parte del turismo que se practica en algunos sectores de la comuna (zona cordillerana) es, realmente, un turismo de aventura y ecoturismo, la llegada masiva de visitantes, a partir de los años ' 90 , puso en peligro las condiciones potenciales de Paihuano para el turismo centrado en la naturaleza;

b) la llegada masiva de turistas, en vez de ser un aporte económico a la comuna, atenta contra el principal atractivo de la zona: su tranquilidad como sitionatural y como zona rural. Esto sólo es posible experimentarlo en temporada baja;

c) para que las autoridades puedan tomar medidas que generen un sistema de manejo y control de la llegada de turistas y preparación del territorio para absorber demanda de servicios turísticos, hacen falta estudios e investigaciones que midan los impactos del turismo en la comuna, bien como las relaciones (tensiones) que pueden producirse entre agricultura y turismo. La creación de la Cotase y la ejecución del proyecto Guarda-Río son, en este sentido, hechos destacables. La elaboración del presente modelo pretende ir también en ese sentido;

d) finalmente, la comunidad y la autoridad local reconocen la importancia de la actividad turística y ven en ella una alternativa que, si se maneja adecuadamente, puede ser sustentable para la comuna.

\section{Referencias Bibliográficas}

CAMPOS, C.; CORTÉS, P.; MONTAÑO, A. 2000. Diseño del proyecto de investigación: evaluación de la gestión turística de la comuna de Paihuano.... La Serena: Universidad de La Serena.

CARRILLO, M.; GONZÁLEZ, J. M. 2001. Un modelo integral de desarrollo de una zona turística. Facultad de Ciencias Económicas. Islas Canarias, España: Universidad de La Laguna.

I. Municipalidad de Paihuano. Análisis y estadísticas. 2000. Documento elaborado por el Departamento de Turismo, Paihuano.

I. Municipalidad de Paihuano. Plan de desarrollo comunal (PLADECO). 2000. Paihuano.

MANNING, E. 1998. Turismo: ¿Dónde están los límites? In Revista RUTA, La Serena: Universidad de La Serena, $n \bullet 2$.

ORGANIZACIÓN MUNDIAL DEL TURISMO - OMT. 2001. Apuntes de metodología de la investigación en turismo. Dirección: Amparo Sancho Perez. Madrid: OMT.

1999. Guia para administradores locales. Desarrollo turístico sostenible. Madrid: OMT. 
1997. Guía práctica para el desarrollo y uso de indicadores de turismo sostenible. Madrid: OMT.

RIVAS, H. 1998. Los impactos ambientales en áreas turísticas rurales y propuestas para la sustentabilidad. Gestión Turística, Valdivia: Universidad Austral de Chile, no 2/98.

SERVICIO NACIONAL DE TURISMO - SERNATUR. Dirección Nacional Santiago. 2003. Secciones de estadisticas y noticias. <http://www.sernatur.cl>. Dirección Regional Coquimbo, La Serena. 1999a. Estudio del gasto turístico. 1999b. Plan maestro de desarrollo turístico para la región de Coquimbo. 1997. Plan de Desarrollo turístico de la comuna de Paihuano.

UNIVERSIDAD DE LA SERENA. 2001. Taller a líderes comunitarios en la comuna de Paihuano. (Documento de trabajo.)

Recibido en 14/02/2003.

Aprobado en 27/04/2003. 